\title{
Japanese Psychiatric Brain Bank and Human Right
}

\author{
Keiko Ikemoto* \\ Department of Psychiatry, Iwaki Kyoritsu General Hospital, Iwaki, 973-8555, Japan
}

\begin{abstract}
Brain bank is an indispensable research resource for pathophysiological studies of mental illnesses. In Japan, a national law for autopsy and dead body storage (1949) has been applied to manage brain bank, though this law is said to need some amendment for research use of human brain materials. In history of Japanese psychiatry, problems of Utsunomiya Hospital, which continued from 1960's and manifested in 1987, caused serious incredulity on psychiatric research and psychiatric medicine. There has been tremendous illegality including autopsy performed by "no" autopsy-qualified individuals such as nurses in Utsunomiya Hospital. In 1987, the Law of Mental Health was amended in Japan in order to protect patients' human right. Recently, following the Great East Japan Earthquake and nuclear disaster in 2011, Fukushima Psychiatric Brain bank, established in 1997, continued its activity in spite of lacking an autopsy-qualified researcher among them. This illegality has been concealed for approximately 2 years. Some problems of human right which have been infringed by brain researchers of psychiatric brain bank and of post-mortem brain studies of psychiatric illnesses in Japan are described.
\end{abstract}

Keywords: Ethico-legal problem; Human right; Brain research; Autopsy qualification; Research resource

\section{Introduction}

Brain research using postmortem brains has been pivotal to explore pathogenesis of neuropsychiatric diseases. Many of ambitious researchers have engaged in postmortem brain study, and have successfully revealed mysteries of human brains. For this, brain banks, i.e. research resources of postmortem brains, have had important roles, though there have not yet been universal consensus in ethics and law in running brain banks [1].

Human tissue and Bio-banking is a complex field and running brain banks needs to abide by several golden standards in order to avoid pitfalls in basic research: 1) A donor system in which informed consent is granted for the use of the samples for scientific research, including genetic analysis and access to medical records, 2) Rapid autopsy system, 3) Compatibility of protocols for procurement, management, handling and storage, 4) A generally accepted consensus on diagnostic criteria, 5) Quality control, 6) Abiding by local/international legal and ethical guidelines for work with human material, 7) Proper safety procedures [2].

However, in Japan, as an Asian county, establishment of brain banks is far delayed in comparison with US and European countries. The present article focuses on some important problems of human right that have disturbed progress of brain science using post-mortem brains of patients with mental illnesses, and establishment of psychiatric brain banks in Japan.

\section{Ethico-Legal Problems}

In Japan, autopsy and brain storage has been allowed in some limited situations or individuals, which has been indicated by a national law. According to this law of autopsy and human dead body storage (1949), autopsy and dead body storage should be done by officially autopsy-qualified individuals assigned by the Ministry of Health, Welfare and Labor in Japan, or by professors and associate professors of departments of anatomy, legal medicine, and pathology. In Japan, under this law, autopsy qualification can be submitted only by medical doctors or dentist assigned by the Ministry of Health, Welfare and Labor in Japan. Some professors or associate professors of anatomical department, who are autopsy-qualified do not have qualification of medical doctor (MD) or dentist. Thus, in Japan, human brain samples have been far accessible for officially autopsy-qualified researchers, the number of which is limited approximately 500 .

Further, being different from US, Japanese system has not yet allowed legal autopsy brains to be used by researchers other than the field of legal medicine. This has long been another obstacle for researchers in the field other than legal medicine, because control samples in pathophysiological studies in order to compare pathological findings with healthy ones are hard to obtain in autopsy other than legal medicine. For this, needs of some amendment of Japanese law for autopsy and dead body storage has been mentioned in view point to promote brain research and establishing brain banks. Nevertheless, this problem remains unsolved.

\section{Problems of Utsunomiya Hospital and of Human Right}

In history of Japanese psychiatry, problems of Utsunomiya Hospital (1960's ), manifested in 1983, caused serious incredulity to psychiatric research and psychiatric medicine. It was reported that 2 patients died due to violence of nurses, however, in addition, numerous illegality, violence, and acts with infringement of human right [3] were also reported. It was known that autopsy had been done by "no" autopsyqualified individuals such as nurses of Utsunomiya Hospital. Psychiatric department of Tokyo University had ties up with Utsunomiya Hospital, and published numerous medical articles by using data obtained from psychiatric patients of this hospital [4]. Severity of these problems remain to influence on activities in the field of Japanese Biological Psychiatry, even after amendment of the Law of Mental Health in 1987 aimed at protecting patients' human right.

*Corresponding author: Keiko Ikemoto, Department of Psychiatry, Iwaki Kyoritsu General Hospital Iwaki, Fukushima, 973-8555, Japan, Tel: +81-246-267-3151; Fax: +81-246-27-2148; E-mail: ikemoto@iwaki-kyoritsu.iwaki.fukushima.jp

Received December 15, 2014; Accepted March 03, 2015; Published March 09 2015

Citation: Ikemoto K (2015) Japanese Psychiatric Brain Bank and Human Right 18: 260 doi: 10.4172/1994-8220.1000260

Copyright: (c) 2015 Ikemoto K. This is an open-access article distributed under the terms of the Creative Commons Attribution License, which permits unrestricted use, distribution, and reproduction in any medium, provided the original author and source are credited 


\section{Men's Ambition and Women Abuse in Japan}

Under the situation that Japanese law of autopsy and brain storage makes human brain samples far accessible for officially autopsyqualified researchers from the Ministry of Health, Welfare and Labor in Japan, ethico-legal limitation in using human brain samples tends to be readily ignored by researchers' ambition. Moral dilemmas in the issues of secondary use of data, data sharing and security may also be related.

FukTushima Psychiatric Brain bank is the first systematic psychiatric brain bank built in 1997, which applied registration advance to death of individuals who wish to donate the brains to this brain bank. This brain bank had been believed to be the one whose ethico-legal 6 system was well arranged.

However, in recent international congress, such as the World Federation of Biological Psychiatry (WFSBP) in Kyoto, Japan, in 2013, it was noted that some Japanese researchers managed the Fukushima Psychiatric Brain bank as if they had an autopsy-qualified researcher among them, though they had already expelled the one from their research group following the Great East Japan Earthquake and nuclear disaster [5]. This illegality has been concealed for about 2 years. To make the matter worse, a man MD researcher, who was a graduate student during the period when the autopsy-qualified woman MD researcher participated in the Fukushima Psychiatric Brain bank, gained the WFSBP congress prize, in spite of his illegality. Surprisingly, most researchers did not regard this situation as a serious matter, or pretended to be so.

Women researchers rather than men researchers are more likely to fall the victim to academic harassment caused by men's excessive ambition. In Japan, as an Asian country, women's social status is relatively low compared with that in US and European countries. Women abuse that exists commonly in Japanese societies, is usually concealed. Recent increase of female students in university medical faculty has lead to reveal the problems from the side of Japanese women MD. In fact, many of Japanese MD researchers are obliged to have their children out of marital relationship, whose fathers are usually their professors of departments, and/or supervisors. Single mothers who rear children without husbands are no rare. In this situation, ambitious women MD and researchers urge the children's fathers to let the other women MD or researchers expel out from their medical and/or academic field(s). Now, almost all of 7 women professors in psychiatric department in Japanese University have experienced to work as single mothers having more than two children of their supervisors.

It should be noticed that such harassment in Japanese style may be a reason why neuropathological or histochemical research using human brain materials has not yet been advanced, and establishment of Psychiatric Brain banks is delayed. In US and European countries, a great contribution of women researchers of neuropathologists and histochemists in brain science has been well-recognized. For example, Dr. Francine M Benes in Harvard Brainbank, Dr. Mayada Akil in the National Institute of Mental Health (NIMH) Brain bank, Dr. Victoria Arango in Columbia University, New York, Dr. Natalia A Uranova in Russia, Dr. Etienne C Hirsch in Pitié-Salpêtrière Hospital in Paris, and so on [6].

In this context, it is not only Japanese ethico-legal system, but also men's excessive ambition which infringed human right of others including colleague researchers, and caused violation of legal restrictions, and academic and sexual harassment of Japanese style, that has long disturbed progress of brain research and development of Japanese Brain Bank.

\section{Japanese Brain Bank}

\section{Perspective of Japanese psychiatric brain bank}

From a historical aspect, brain research using post-mortem brains of patients with mental illnesses has had serious ethico-legal problems in Japan. The Japanese Society of Biological Psychiatry organized the Committee of Establishment of Brain bank in 2007, aimed at development of Psychiatric brain bank in Japan. Nevertheless, similar problems of 8 infringement of human right of researchers, patients' families, and medical staffs have continued, and the problems have been concealed as if they were seemingly much improved. Whereas, the Japanese Society of Psychiatry and Neurology newly organized the Gender Equality Promotion Committee, and the first meeting was held on the 14th, September, 2013. It would be expected that Japanese academic societies promote gender equality as well as brain research, although it remains unclear whether the harassment of Japanese style would successfully be prevented.

\section{References.}

1. Ikemoto K (2008) "Neural stem cells and Epigenetics" as new strategies for psychiatric research using post-mortem brains: After the 1st Symposium for Brain Bank, 22 October 2006, Fukushima, Japan. Nature 452: 263.

2. Ravid R, Ikemoto K (2012) Pitfalls and practicalities in collecting and banking human brain tissues for research on psychiatric and neurological disorders. Fukushima J Med Sci 58: 82-87.

3. Ikemoto K, Nakamura M (2004) Forced deprogramming from a religion and mental health: a case report of PTSD. Int J Law Psychiatry 27: 147-155.

4. Utsunomiya Byoin Jiken (2015)

5. Ikemoto K (2015) Catastrophe of Japanese psychiatric brainbank. J Psychother Psychol Disor 2: 1.

6. Ikemoto K (2012) Neuropathological research of mental illnesses: "D-cell hypothesis" of schizophrenia. Jpn J Gen Hosp Psychiatry 24: 388-397. 